\title{
Escritura como práctica de sí y escuela rural
}

Paola Andrea Lara Buitrago

Magíster en Educación

Universidad Pedagógica y

Tecnológica de Colombia

paola.lara@uptc.edu.co

https://orcid.org/0000-0003-1975-3371

\section{Oscar Pulido Cortés}

Doctor en Ciencias de la Educación

Universidad Pedagógica y

Tecnológica de Colombia

oscar.pulido@uptc.edu.co

https://orcid.org/0000-0002-3863-5007

\section{Artículo de Investigación}

Recepción: 01 de septiembre de 2019 Aprobación: 10 de diciembre de 2019 https://doi.org/10.19053/22160159.v11.n25.2020.10480

\section{Resumen}

El artículo presenta los resultados del proyecto de investigación Escuela rural y escritura de sí: una experiencia pedagógica, en el que se propone la escritura como experiencia de sí y su interacción para la constitución de sujetos en una escuela rural colombiana. La perspectiva metodológica está ubicada en los desarrollos del momento ético de Michel Foucault quien se plantea como ejercicio de sí en el pensamiento e involucra la experiencia de los sujetos y sus posibilidades de transformación. La estructura teórica está delimitada en cuatro categorías: escritura como práctica de sí, niño-escritor, trabajo con la ruralidad y la lectura como ejercicio espiritual. En los resultados y en la discusión se describen los ejercicios de escritura que se desarrollaron como propuesta práctica en los que sus destinatarios interactuaron con las posibilidades de escribir no solo códigos lingüísticos sino que también 
tuvieron la experiencia de la escritura como forma de constitución de sí mismos. Para finalizar, el artículo aborda algunas conclusiones que destacan la escritura como un ejercicio que se aleja de la instrumentalización y de la decodificación del lenguaje.

Palabras clave: escritura, educación rural, infancia, enseñanza de la filosofía, Foucault

\section{Writing as self-practice and rural school}

\section{Abstract}

The article presents the results of the research project "Escuela rural y escritura de sí: una experiencia pedagógica" (Rural school and writing of oneself: a pedagogical experience), in which writing is proposed as an experience of oneself and its interaction for the constitution of subjects in a Colombian rural school. The methodological perspective is based on the developments of the ethical moment in Michel Foucault who proposes himself as an exercise in thought involving the subjects' experience and their possibilities of transformation. The theoretical structure is divided into four categories: writing as self-practice, child-writer, work with rurality and reading as a spiritual exercise. The results and the discussion describe the writing exercises that were developed as a practical proposal in which the recipients interacted with the possibilities of writing, not only linguistic codes, but they also experienced writing as a way of constituting themselves. Finally, the article addresses some conclusions that highlight writing as an exercise that moves away from the instrumentalization and the decoding of language.

Keywords: writing, rural education, childhood, philosophy teaching, Foucault

\section{L'écriture comme pratique de soi et l'école rurale}

\section{Résumé}

L'article présente les résultats du projet de recherche "Escuela rural y escritura de sí : una experiencia pedagógica" (École rurale et écriture de soi : une expérience pédagogique), dans lequel on propose l'écriture comme une expérience de soi et son interaction pour la constitution des sujets dans une école rurale colombienne. 
La perspective méthodologique s'inscrit dans les développements du moment éthique de Michel Foucault qui se propose lui-même comme un exercice de soi dans la pensée et implique l'expérience des sujets et leurs possibilités de transformation. La structure théorique est divisée en quatre catégories : l'écriture comme pratique de soi, enfant-écrivain, le travail avec la ruralité et la lecture comme exercice spirituel. Les résultats et la discussion décrivent les exercices d'écriture qui ont été développés en tant qu'une proposition pratique, dans lesquels les destinataires ont interagi avec les possibilités d'écriture, non seulement les codes linguistiques, mais ils ont également expérimenté l'écriture comme une manière de se constituer eux-mêmes. Enfin, l'article aborde certaines conclusions qui soulignent que l'écriture est un exercice qui s'éloigne de l'instrumentalisation et du décodage du langage.

Mots-clés : écriture, éducation rurale, enfance, enseignement de la philosophie, Foucault

\section{Escritura como prática de sim e escola rural}

\section{Resumo}

O artigo apresenta os resultados do projeto de pesquisa Escola Rural e escritura de sim: uma experiência pedagógica, na qual é proposta a escritura como experiência de sim e sua interação para a constituição de sujeitos numa escola rural colombiana. A perspectiva metodológica está localizada nos desenvolvimentos do momento ético de Michel Foucault, quem se considera como exercício de sim no pensamento e envolve a experiência dos sujeitos e suas possibilidades de transformação. A estrutura teórica está delimitada em quatro categorias: escritura como prática de sim, criança-escritor, trabalho com a ruralidade e a leitura como um exercício espiritual. Nos resultados e discussão descrevem-se os exercícios de escritura que foram desenvolvidos como proposta prática em que seus destinatários interagiram com as possibilidades de escrever, não só códigos linguísticos senão que também tiveram a experiência de a escritura, como forma de constituição de sim mesmos. Finalmente, o artigo aborda algumas conclusões que destacam a escritura como um exercício que se afasta da instrumentalização e decodificação da linguagem

Palavras-chave: escritura, educação rural, infância, ensino da filosofia, Foucault 


\section{Introducción}

Este artículo de investigación es producto del proyecto: $L a$ escritura: creatividad y experiencia en la escuela rural.SGI 2307 DINUPTC. En se trata la importancia de la escritura en las instituciones educativas y en las prácticas tradicionales. La escritura está orientada al cumplimiento del objetivo principal de la educación inicial; la alfabetización, que se convierte en requisito para la promoción escolar. Los múltiples efectos que genera la escritura se encuentran asociados al desacuerdo o disgusto, al producir algún escrito por parte del estudiante, en otras palabras, la actitud que se muestra al momento de proponer en las aulas algunas prácticas escriturales; de igual forma, estan asociados a la existencia de una barrera creativa que no posibilita espacios de invención y limita las prácticas de escritura a repeticiones de palabras o transcripción de textos.

Por otra parte, y es un caso muy común, las acciones escolares privilegian la oralidad y los estudiantes se acostumbran a la transmisión de la palabra y no, al ejercicio escrito. En esta perspectiva, los niños y niñas de la escuela presentan dificultades en el proceso de aprendizaje de la escritura y para escribir con sentido y significado. Además, encuentran que los escritos desconocen su contexto y los perciben ajenos a sí mismos.

La escritura no se reduce únicamente al sujeto que escribe o el escritor. La escritura permite identificar otras dimensiones: el sujeto que escribe, lo escrito, y el lector; estas tres dimensiones representan el acto de escribir, en sus maneras de actuación. De este modo, surgen preguntas en tres niveles de complejidad: en el primero de ellos, se pregunta por el sujeto que escribe y puntualiza sobre interrogantes para conocer su posición, es decir, el escritor se interroga acerca de: ¿cómo se constituye él cuando escribe?, ¿qué tipo de manifestaciones suscita el acto de escribir, ¿la escritura es transformación de la vida? y si ¿̇en el acto de escribir se encuentra un conocimiento de sí mismo o es una descripción de acontecimientos?

En el segundo nivel, se pregunta por el escrito: si este se aproxima al conocimiento dela función que cumpleen determinados escenarios, como la escolaridad y la academia, donde es posible distinguirlo en resultados de alfabetización, medio de circulación de información, publicación de resultados, sistematización de experiencias, requisitos de promoción y divulgación de investigaciones. 
En el tercer nivel de las preguntas, se inscribe al sujeto que lee, quien a partir del escrito escucha lo que el otro dice, se conecta con lo que se escribe y conversa con el autor y distingue, entre líneas, al escritor.

La inquietud que desarrolla en este artículo es propiamente por el sujeto escritor, en su proceso de configuración en la escuela rural. El interés es indagar la escritura como práctica de sí, que responde a los ejercicios de autocontrol o dominio del sujeto sobre sí mismo y su relación con la constitución de la vida; de igual forma, como técnica que transforma la existencia del sujeto. Esta dualidad de concepciones se aborda desde la noción de escritura de sí analizada por el filósofo francés Foucault (1998, 1999, 2002, 2013, 2012a, 2015) y sus derivaciones conceptuales. Los desarrollos teóricos y la apropiación de las concepciones surgen a partir de los proyectos de investigación ${ }^{1}$ los cuales, en sus primeras fases, se fundamentaron en la búsqueda teórica del autor mencionado, y de algunas nociones que se intentan relacionar en este artículo.

\section{Problematización}

La necesidad de idear y desarrollar un proyecto de investigación en la escuela rural (Morales \& Pulido, 2019) centrado en la escritura, parte de un desconocimiento existente sobre el sujeto que escribe en relación con su texto y contexto no solo inmediato, sino personal e íntimo. De ahí la necesidad de investigar una forma-problema que convoque a la escritura propia, despierte interés y que no sea desconocida para el estudiante, pues suscita un encuentro en la palabra, el texto, la emoción y los sentimientos. Así los niños y niñas encuentran en estos textos posibilidades de experiencia en lo que escriben, y a su vez, establecen conexiones con la realidad y la cotidianidad de la vida.

A través de esta forma-problema se debe lograr un distanciamiento o diálogo crítico con las prácticas tradicionales que se normalizan en la escuela rural para el aprendizaje de la escritura, tales como el uso de las cartillas y las guías de escuela nueva, que en la mayoría de las ocasiones producen prácticas de repetición, transcripción o reproducción de expresiones. En este campo

1 La escritura pedagógica: modos de hacer experiencia en la escuela rural UPTC-SGI 2343 y La escritura: creatividad y experiencia en la escuela rural UPTC-SGI 2307 
problémico, el proyecto responde a la pregunta: ¿de qué manera la escritura de sí configura el reconocimiento de estudiantes de la escuela rural como sujetos y contribuye a su proceso de constitución?

El propósito de la investigación fue reconocer la escritura de sí ${ }^{2}$ como posibilidad de transformación y encuentro de los sujetos de la escuela rural consigo mismos, a través de la escritura como el acto que permite la transformación. El objetivo general se apoya en indagar la importancia que ocupa la escritura de sí en la práctica escolar y la relación que se obtiene en la configuración del estudiante durante el proceso formativo, la conexión y reconocimiento del sujeto y sus concepciones con las formas en las que la escritura habita la escuela; un segundo aspecto consiste en posibilitar escenarios de formación dirigidos a los ejercicios de escritura para que fortalezcan, en los estudiantes, el conocimiento sobre sí mismos, a partir de ejercicios de escritura y cómo estos consolidan la constitución de sujetos. Por último, se propuso reconocer los procesos de subjetivación, desde la escritura de sí, en la interacción con el maestro, el investigador y los niños y niñas involucrados en la investigación.

Las finalidades y las intenciones de la investigación fueron el posibilitar, en los sujetos de la escuela rural, un reconocimiento de sus potencialidades a partir de prácticas de escritura, además de permitir al maestro su interacción, en términos de la creación para la modificación de las prácticas tradicionales implementadas en la escuela. De igual modo, se buscó contribuir en la construcción de un campo teórico sobre escrituras alternativas, puesto que la categoría de escritura de sí aún no ha sido lo suficientemente teorizada en el espacio de la escuela primaria. Otro aspecto que se presenta como aporte del trabajo de investigación se relaciona con el hecho de que la escritura guarda una intención con respecto a la formación de maestros investigadores en filosofía e infancia y educación rural.

El artículo se organiza en cuatro apartados: metodología, fundamento teórico, resultados y discusión y conclusiones.

2 La escritura de sí se comprende en este trabajo como un ejercicio espiritual en la perspectiva de la Antigua Grecia - escuelas helenísticas romanas, estoicas y epicureistas- donde escribir no solo es un acto de codificación de lenguaje, sino una experiencia de vida, un arte de reconocerse y una forma de esculpir el carácter y constituir un ethos. 


\section{Metodología}

La perspectiva metodológica se despliega en dos planos concomitantes y yuxtapuestos: en el primero, como enfoque de la investigación, se asume una mirada filosófica y se opta por el ensayo (Espinel \& Pulido, 2017a) o prueba modificadora del pensamiento en el trabajo crítico sobre sí mismo (Foucault, 2012b). Se asume el giro metodológico en la última etapa de su producción académica (Espinel \& Pulido, 2017; Pulido, 2017). Adicionalmente, se plantea que este ejercicio de pensamiento se identifica en la transformación del sujeto que se problematiza y en las experiencias que permiten movilizarse y pensarse de otras maneras; igualmente, en el enfoque que asume una particular mirada sobre los sujetos y sus posibilidades de constitución, a través de prácticas de libertad (Foucault, 1999). Dichas prácticas se encuentran en la escritura, un intersticio en el cual las acciones, las formas de ser y sentir confluyen y se recrean.

Así es como los sujetos involucrados en este doble juego de ensayar y constituirse se acercan a la escritura de otra manera, en otros sentidos, para modificar el horizonte de lo conocido y lo realizado en la educación instrumentalizada y mercantilizada. De otra parte, se asume como procedimiento la revisión documental de fuentes teóricas en la obra de Foucault, el rastreo conceptual con el abordaje de la categoría escritura de sí y la tematización de sus enunciados. Dentro de los instrumentos utilizados se encuentran las fichas temáticas y analíticas, a partir de las cuales se realizó un estado de la cuestión de investigaciones contemporáneas en revistas especializadas sobre la escritura de sí, y se obtuvieron las siguientes tendencias: a) entre el cuidado y la estética de la existencia; b) la escritura de sí como producto de subjetividad y experiencia; c) la materialización de la práctica que reúne los avances y experiencias en la escolaridad o en contextos formales de educación, inspirados a través de la escritura de sí, y por último; d) la escritura de sí entre formas y modos, como otras experiencias de escritura que permiten hacer el diálogo, entre la categoría y las diferentes formas de expresión.

El segundo plano, se centró en la intervención de la escuela y se hicieron análisis por medio de algunas herramientas de la investigación-acción. Se recurrió a técnicas de análisis distribuidas en dos momentos: en el primer momento fueron utilizadas en la intervención: la observación participante, las entrevistas, los 
ejercicios y los consentimientos informados, para cumplir con las consideraciones éticas de la investigación.

En el segundo momento, las técnicas aprovechadas para el trabajo documental fueron los escritos, producto de otras experiencias en la escuela ${ }^{3}$ (Cely, Espinosa \& Suárez, 2016; Hernández \& Hernández, 2012; Pulido, Suárez \& Espinel, 2017; Suárez, Mariño \& Espinel, 2001) y los cuadernos de escritura que se destinaron para la consecución de los ejercicios aquí planteados. Para el análisis de información se recurrió a una triangulación entre la observación participante, la observación de lo que se realizaba en los talleres, las entrevistas con el maestro y los productos o escritos que consolidaron los niños. Este análisis se organizó a partir de tres categorías: la escritura de sí, entre el cuidado y la estética de la existencia; la segunda categoría se define a partir de la escritura de sí entre modos de subjetivación y experiencia, y por último; la escritura de sí entre formas y modos. Las anteriores categorías se articulan con la revisión teórica y el estado de la cuestión.

\section{Referentes teóricos}

La perspectiva teórica tuvo en la obra de Foucault el principal soporte, especialmente en las producciones académicas comprendidas entre los años 1980-1984, tiempo en el que el énfasis de su trabajo giró alrededor del sujeto. Allí, el filósofo opta por el sujeto y su relación con la ética (Lara, 2017). La ética es concebida como la relación del sujeto consigo mismo, a través de principios y técnicas que permiten la modificación, transformación y configuración de vida como obra de arte $^{4}$. Este tránsito resume dos principios filosóficos que se privilegiaron en la época griega y romana: el conócete a ti mismo y el cuidado de sí. Este segundo principio, representó para la investigación, la mayor parte del

3 Los proyectos de investigación desarrollados en la escuela rural corresponden a: Filosofía e infancia: investigación y posibilidades de innovación en la escuela rural, UPTC SGI 1616; El campamento filosófico I, II, III, La escritura pedagógica: modos de hacer experiencia en la escuela rural UPTC-SGI 2343. La escritura: creatividad y experiencia en la escuela rural UPTC-SGI 2307, Así mismo, se resaltan los artículos producto de proyectos en la escuela rural.

4 En la perspectiva de Schmid, Foucault retoma el pensamiento de Nietzsche, al hablar del arte de vivir, al establecer que el yo puede formarse y transformarse, a partir de ejercicios personales y de experiencias independientes de las formas determinadas y dadas por la moral. Ahora bien, la relación del arte con la vida es la posibilidad de comprender la vida como espectáculo y crearse como una obra. 
análisis del sujeto en relación consigo mismo, en la búsqueda de la configuración de la verdad y en el cómo él se ha constituido, a partir de esas verdades. De modo que es un sujeto de veridicción que atiende a algunas formas o actividades sobre sí mismo, las cuales permiten hacer una vinculación entre la verdad y los modos de subjetivación.

En este sentido, se plantean cuatro categorías que desarrollan el sustento teórico de la investigación, a saber: escritura como práctica de sí, niño-escritor, trabajo con la ruralidad y sus implicaciones y la lectura como ejercicio espiritual. Estas categorías permiten el despliegue del fundamento teórico para la comprensión de las relaciones halladas en el trabajo con la escuela rural.

\section{Escritura como práctica de sí}

Michel Foucault, en el momento ético, analiza las escuelas filosóficas grecorromanas ${ }^{5}$ y ubica allí la cultura del cuidado, la cual da cuenta de la ética no como un conjunto de preceptos y prescripciones morales, sino detallada desde el mismo sujeto y la manera cómo modifica su pensamiento y conducta, con la finalidad de hacer de su vida el objeto que configure su existencia. El alcance de esta vida necesita de técnicas y ejercicios que guían su fundamento. Por eso las técnicas de sí, correspondientes a la escritura, la lectura y la meditación, posibilitan en el sujeto el reconocimiento de sí mismo y su transformación, a través de la ascesis.

La ascesis de la práctica de sí de la época que nos ocupa, se trata de reunirse consigo mismo como fin y objeto de una técnica de vida, un arte de vivir, se trata de reunirse consigo mismo con un momento esencial que no es el de la objetivación de sí en un discurso de verdad, sino el de la subjetivación de un discurso de verdad en una práctica y un ejercicio de sí, sobre sí mismo. (Foucault, 2002, p. 317)

La escritura es uno de los procesos de la ascesis (Lechuga, 2007); además el gnothi seauton -conocimiento de sí mismo-y la epimeleia heatou - cuidado de sí- designada por el conjunto

5 La civilización de la Grecia antigua de los siglos IV a.C hasta II y III d.C, se caracterizó por ser una cultura principalmente oral. Siglos después, la inserción de la palabra escrita fue gracias a algunos filósofos y pensadores, quienes eran privilegiados en aprenderla, a cambio de pago. La escritura se consideró como una práctica de carácter privado. 
de condiciones de espiritualidad y de transformación de sí son principios necesarios para la formación del sujeto mismo, que está direccionado hacia la búsqueda de verdad y las formas del acceso a la misma. En la Antigüedad se consideraba que los modos de pensar y practicar la vida se articulaban con el acceso a la verdad y las transformaciones para conseguirla, a través de la reflexión y modificación de las prácticas y experiencias de los sujetos (Morresi, 2005).

En el proceso de subjetivación, donde el sujeto se constituye a través de prácticas y discursos, la escritura se anida en la práctica de sí mismo e indaga por su reflexión sobre los actos y en la proyección de posibilidades de actuación o de construcción del estilo de vida. En efecto, la escritura tiene una cierta dualidad al servir en dos escenarios, uno a nivel personal y otro a nivel social o colectivo. Como ejemplo, en los compendios de Sneca (citado por Foucault, 2002) ${ }^{6}$, se identifica esta práctica escritural para sí mismo y los otros. En la correspondencia amistosa, se descubre la posibilidad de reflexión del sujeto, en tanto se regresa a revisar la experiencia, es decir, es el paso para retornar sobre sí mismo que contempla el examen de lo sucedido y la comprensión al respecto.

A nivel social, comprende la búsqueda y lectura de consejos para el conocimiento de la vida o las posibilidades de acción. Igualmente, en este recurso de la escritura de cartas se representa un discurso impreso de alguna veracidad a quien lo lee, de modo que la escritura evidencia manifestaciones de verdad y con carácter de confesión para otro. Este acercamiento de juegos de verdad, Foucault (1999) lo plantea como:

El ejercicio personal hecho por sí y para sí es un arte de la verdad inconexa o, más precisamente, una manera reflexiva de combinar la autoridad tradicional de la cosa ya dicha con la singularidad de la verdad que en ella se afirma y la particularidad de las circunstancias que al respecto determinan su uso. (p. 295)

Es decir, la enunciación de alguna verdad, de su verdad, de su acción de verdad (Foucault, 2010), modifica la existencia de quien escribe y lee. La escritura refleja el ejercicio de sí y compromete la acción reflexiva en el sujeto. Además comprende un lector que

6 Foucault (2002) estudia los compendios de Séneca como De la tranquilidad del alma, Cartas a Lucilio. 
hace lectura atenta de lo expresado y genera consejos y opiniones que constituyen manifestación de sí (Foucault, 1999), o juegos de verdad, por lo tanto no se piensa solamente en el escritor que escribe para sí mismo, sino que es un escritor que dimensiona aperturas al reconocer el otro, como parte de su escritura, lo que significa que se escribe para alguien indiscutiblemente. Se cuenta con la presencia del lector que me lee, por consiguiente: "escribir es, por tanto, «mostrarse», hacerse ver, hacer aparecer el propio rastro ante el otro.” (Foucault, 1999, p. 300). Así en la correspondencia se evidencia el acercamiento de la escritura y la lectura, técnicas complementarias entre sí.

Otro ejemplo de la escritura, en la época clásica, lo constituyen los libros guía de conducta o hypomnémata. Estos textos funcionaban como directrices para tener en cuenta y constituir estilos de vida. En ellos se podían encontrar "citas, fragmentos de obras, ejemplos extraídos de la vida de personajes más o menos conocidos, anécdotas, aforismos, reflexiones o razonamientos" (Foucault, 2015, p. 362), que apoyaban el trabajo del sujeto sobre sí mismo y le proporcionaban maneras de solucionar problemas o situaciones.

"La escritura aparece regularmente asociada a la «meditación», a ese ejercicio del pensamiento sobre sí mismo que reactiva lo que sabe, vuelve a hacer presentes para sí un principio" (Foucault, 1999, p. 291). Según Lechuga (2007), la diferencia entre ambos es que en los hypomnémata, los fragmentos son materia de reflexión y escritura y la correspondencia comunica técnicas y reglas de vida desde la mirada de uno mismo en los otros.

Toda la "literatura del yo" puede enmarcarse en este amplio marco general de las prácticas del yo. La escritura no es así sino un medio del arte de conducir la propia vida y darle forma, supone un gesto de la existencia y de la configuración de sí. En el ejercicio de esta actividad el individuo se forma, proyecta la posibilidad de su existencia y de su transformación. (Schmid, 2002, p. 282)

La formación del yo se da en ese momento. La escritura se convierte en detonante de existencia y responde a alguna estética y estilo. La escritura como arte de la existencia suscita el trabajo consigo mismo, de transformación y distanciamiento de los regímenes apropiados para reflexionar y encontrarse con otras posibilidades y alternativas de pensamiento. 
La estética de la existencia trata de la transformación del individuo relacionada con la verdad y el saber:

El sujeto que se comprende como obra de arte, no tiene intención de descubrirse a "sí mismo" en toda su verdad y pureza, ni de liberar el "ser auténtico" del hombre, sino de inventarse y de realizar un trabajo sobre sí mismo. (Schmid, 2002, p. 271)

La escritura percibida desde el cuidado, los ejercicios espirituales, el trabajo sobre sí mismo, el arte de vivir y la ascesis contribuyen a la compresión del sujeto en su experiencia. Aunque la escritura persiga la transformación singular, no desconoce el lugar donde se produce. Los espacios donde se comparte esta técnica se describen como sitios contingentes susceptibles de habitar y campos de transgresión que provocan distanciamiento del pensamiento.

La escritura como práctica personal y ejercicio ascético se describía con funciones ethopoiéticas, cercana a la meditación y necesaria de la reflexión para responder a la realidad en situaciones problemáticas, o sea, la escritura al igual que otras prácticas de sí, precisan de ejercicio y del entrenamiento de sí por sí mismo en especial al pensamiento. "La escritura constituye una etapa esencial en el proceso al que tiende toda áskesis: a saber, la elaboración de discursos recibidos y reconocidos como verdaderos en principios racionales de acción." (Foucault, 1999, p. 292). La escritura antigua de la cultura griega y romana se complementaba con la lectura y la escucha, en el ejercicio de meditación detonados desde la experiencia del sujeto el conocimiento de sí mismo y con atención al constante cuidado con el objetivo de la transformación. ${ }^{7}$

\section{Niño-escritor}

El autor aparece como una figura que se traslada a diferentes escenarios de circulación y actuación, que dependen de ciertas fuerzas, las cuales posibilitan que asuma la función de autor, pero esas fuerzas son fluctuantes y resultado de condiciones que permiten que algunos discursos se conozcan e identifiquen más que otros. Según Foucault (1998) "La función autor es pues característica del

7 Según Pérez (2006), la escritura en los griegos y romanos reunía el dictado, la transcripción, la expresión de sí mismo, lectura vocalizada, lectura pública y discusión. 
modo de existencia, de circulación y de funcionamiento de ciertos discursos en el interior de una sociedad.” (p. 46), en tanto que las condiciones de la sociedad generan también que unas verdades se posicionen más que otras y tengan mayor grado de veracidad, por las formas de legitimación en las que están inmersas: sitio del maestro, comunidades académicas, orientaciones de política, entre otras.

Así mismo, existe una problematización por la autoría, puesto que un escrito representa discursos y su circulación no depende solo de un sujeto, de modo que la escritura, a pesar de provenir de un sujeto, no establece la distinción de quién habla, pues el autor en estos términos toma el lugar de autor para expresar, comunicar, reconocer y configurar un discurso, y tiene en cuenta que los discursos se plantean como el conjunto que moviliza el pensamiento y las maneras de conocer. Por eso, se problematiza al sujeto que escribe, desde la autoría, porque es a través de ella que organiza y dispone de diversos elementos para decir y expresar. A partir de este momento es que el niño en la escuela, en sus procesos educativos, aparece para decir algo a través de su escritura, comunica su verdad en tanto reproducción, modificación o construcción de lo que cree y cómo conduce su vida. Así "El nombre de autor está situado en la ruptura que instaura un determinado grupo de discursos y su modo de ser singular." (Foucault, 1998, p. 46). La verdad consiente la idea de sujeto y, a partir de la misma, se reposiciona como autor.

El niño-escritor ${ }^{8}$ emerge gracias a la articulación con la función autor, en la medida en la que los modos de ser singular son autorreflexivos y de reconocimiento de cada uno. La experiencia toma lugar para ser el sustento que posibilita la escritura, en tanto que la experiencia como reflexión de sí mismo implica la transformación (Lucci, 2013; Lluch, 2013). En la configuración del niño-escritor, la experiencia es necesaria en la escritura, ya que permite que se transite por la subjetividad y el reconocimiento propio, se reafirme un acontecimiento y se provoque un ser distinto y un pensamiento diferente. El niño-escritor complejiza

8 Para esta investigación se ha elegido la categoría niño-escritor referido al sujeto que escribe -niño-y escritor como el ejercicio que el niño hace de su escritura, es decir, el niño que se moviliza sobre el ejercicio de escribir. En la línea de Deleuze, se plantea que el niño deviene escritor y la escritura deviene niño, en la medida en que el devenir implica coexistencia, indiferenciación e indeterminación. 
la función cuando se determina la vinculación de reconocimiento en lugares y tiempos, y aquello que le produce una experiencia se relaciona con su reconocimiento como sujeto, en su necesidad de transformación y autoformación en diferentes acontecimientos (Delory-Momberger, 2006; Demetrio, 2013 \& López, 2015). Más aún, en la escritura ubica el proceso de subjetivación, al articular la producción y la expresión de la verdad como sujeto de veridicción.

Por ello, el niño-escritor produce existencia a los procesos de subjetivación. Se centra en la pregunta por el sujeto, en este caso, por el niño, con el propósito de conocer, explorar y reflexionar sobre su vida, y de encontrar ese sustento para escribir y crear desde sí con pasión (Domingues, 2004). El encuentro de la subjetividad y la creación ubica al niño-escritor en el vórtice, entre el interior y el exterior (Aguilar, 2017; Aquino, 2011; Arias, 2012; Castorina, 2013 \& Scholze, 2007), pues es desde lo íntimo y personal que pasa para comunicar y expresar a otros.

\section{Trabajo con la ruralidad y sus implicaciones}

La escuela rural se representa como el escenario de posibilidades, gracias a ciertas condiciones que transforman la práctica pedagógica. La articulación de la escuela rural y la escritura de sí permite repensar el ejercicio de escribir, desde la técnica y la práctica que evoca la autoformación, conocimiento y cuidado del sujeto. En especial, la ruralidad posee características que son importantes para el desarrollo de propuestas de investigación, en este caso, de escritura para trabajar de otra manera. El escenario rural teje relaciones que apropian y movilizan el paso de sujetos por la escolaridad y, en el planteamiento de acciones educativas que vinculan la tradición, la apropiación y los diálogos, con lo particular y singular de su contexto: la relación con lo agrario, lo ancestral, las costumbres, ciertas formas de ser familia, de trabajar con la tierra, de disfrutar la vida; como también las formas de vulnerabilidad, abandono y discriminación, a las que estas poblaciones han sido sometidas.

Las implicaciones de la ruralidad, en el ejercicio de escribir, se complementan con la intensa relación que los estudiantes comparten con la naturaleza y el entorno, pues estas dos características originan e integran el escribir y el leer, a partir de ello (Loponte, 2006; Silveira, Nascimento \& Galindo, 2016). Así 
mismo, la escuela en la ruralidad se convierte en un centro donde los acontecimientos generan experiencias en el sujeto y en los sujetos, pues permite construir, determinar, criticar, resistir o distanciar formas de vida y existencia del estar en la escuela. Adicionalmente, la misma escuela despierta emociones, sensaciones y expresiones para escribir, desde la experiencia vital y las posibilidades de ser de otras maneras.

La ruralidad convoca a aproximaciones a la pregunta por la necesidad de la escuela en escenarios, en ocasiones olvidados, apartados y descentralizados y, además, lleva a la pregunta por la situación de la escuela rural y las potencialidades que se despliegan para problematizar, transformar, deconstruir y reflexionar, en torno a espacios de formación, expresión e interacción, bajo el hecho de que las condiciones de existencia de esta escuela posibilitan otro tipo de prácticas y discursos importantes para repensar la experiencia de ser maestro, el lugar del sujeto que aprende y las formas de enseñanza.

\section{Lectura como ejercicio espiritual}

La escritura se encuentra con la lectura, deviene lectura y la lectura deviene escritura. Según Deleuze (1993) la escritura es inseparable del devenir, en el sentido en que el devenir no es una identificación, sino una zona de vecindad: se es diferente, pero se coexiste. Lectura y escritura coexisten, puesto que constituyen una zona que prioriza el entre, es decir, la relación, el encuentro, consiste en "un paso de vida que traviesa lo vivible y los vivido" ( $\mathrm{p}$. 11). Lectura y escritura son, a su vez, técnicas y prácticas que se identifican en la civilización grecorromana. La lectura junto con la escucha se constituyen en las otras técnicas de vida o tekhne tou biou que permiten la relación del sujeto consigo mismo y se dirigen a la transformación de la existencia. En la educación, se distinguen la lectura y la escritura como procesos complementarios y de igual importancia, de modo que no se deja de lado el hablar de lectura en este trabajo de escritura. A proposito de la lectura en tanto ejercicio espiritual, según Hadot (2006):

La palabra «espiritual» permite comprender con mayor facilidad que unos ejercicios como estos son producto no solo del pensamiento, sino de una totalidad psíquica del individuo que, 
en especial, revela el auténtico alcance de tales prácticas: gracias a ellas el individuo accede al círculo del espíritu objetivo, lo que significa que va situarse en la perspectiva del todo. (p. 24)

Desde el ejercicio espiritual, en la perspectiva de Hadot, sucede en el retorno y regreso del sujeto hacia sí mismo. La lectura convoca a meditar, a guardar tiempo y tranquilidad para leer, ya que es importante dejarse invitar por el comentario del autor, por su escritura y detenerse a escuchar. Este ejercicio espiritual, según Hadot (2006), es uno de los más complejos, en razón de comprender y vivenciar aquello que está en el texto y en la necesidad del repliegue del sujeto sobre sí mismo, en relación con la escucha, la actividad mental y liberación de todo tipo de preocupaciones. De manera que la lectura no es únicamente la decodificación del lenguaje escrito, por el contrario, se resume en un momento para volver sobre sí, hacer una experiencia durante la lectura y retornar hacia el alma para generar una disposición al momento de leer, en el que la tranquilidad, la libertad y el dominio del sujeto consigo mismo se fortalecen el autorreconocimiento y formación de sí mismo en la búsqueda del ejercicio de su pensamiento. El niño deviene autor.

\section{Resultados y discusión}

En la antigüedad, las técnicas de constitución de la subjetividad y su ejercicio se desarrollaban únicamente para los adultos, sin embargo, la intención en este trabajo es pensar la escritura de sí y sus tecnologías, para niños y niñas escolares, con la idea de que en ella se distinga un ejercicio, espiritual, filosófico y literario que permita la conexión con la vida y con la escuela rural, pues al entenderla como ejercicio, se intenta afectar la comprensión del niño de sí mismo, quien reconoce su configuración como sujeto, se manifiesta y establece relaciones alrededor de la familia, del cuidado y de la experiencia como fundamentos de su vida. Adicionalmente, la escritura pensada en la técnica y la práctica se encamina en la consideración de la filosofía como ejercicio de pensamiento, desde la perspectiva de constitución de sujetos que se comprometen a vivir de distinta manera la escritura en la escuela.

Los ejercicios de sí se estructuraron en dos momentos: el primero estuvo constituido por ejercicios que tuvieron el objetivo 
de hacer un diagnóstico-valoración ${ }^{9}$ del estado de la escritura de los estudiantes. Posteriormente, se utilizaron algunas técnicas de invención que posibilitaran la creación y detonaran la creatividad en los estudiantes. Las dos actividades iniciales fueron la escritura natural y un ejercicio denominado componer otro final. Para auscultar si los niños ya estaban en una etapa de proceso escritor convencional o si todavía no la pasaban. Esto influyó en los posteriores ejercicios de escritura de sí, donde se definieron y se tuvieron en cuenta los niños que escribían convencionalmente. Los restantes ejercicios, tales como el binomio fantástico, la construcción de adivinanzas y los viejos juegos, se tomaron y adaptaron de las técnicas de invención de Rodari (1973).

Los ejercicios de escritura de sí se consideraron en el segundo momento de la intervención: un escrito colectivo, el autorretrato, las autobiografías, la correspondencia y el diario de experiencias con inspiración en la cultura grecorromana estudiadas por Foucault y Hadot. En lo que sigue del escrito se describen las técnicas y los resultados retomados de la experiencia.

En el cuento colectivo se pueden definir algunas aproximaciones y relaciones de las categorías teóricas inspiradas en las escuelas grecorromanas. Se inicia por un cuidado de lo otro. Al escribir entre varios estudiantes se cuestiona, desde la inquietud del que se escribe, un cuidado que va a llegar a otras personas, la ocupación de cómo inspira la creación de mi escrito a la creación del otro compañero o a la continuación de su escrito, y la preocupación que consiste en cómo me preparo para escribir. Las prácticas de escucha también son identificadas en el cuento colectivo y, para que esa práctica de escucha se desarrollara, junto con los niños se crearon algunos principios propios tales como: el silencio, la disposición corporal y la atención.

El cuento colectivo emerge en espacios de libertad que son resistentes a dominios como los del maestro, porque no le exige que esté pendiente de la tarea, sino que quien escribe se encuentra en su proceso de creación, bajo el dominio de sí mismo y da lugar a

9 En este trabajo, el diagnóstico se comprende como el acercamiento de los investigadores para leer la relación de los niños y niñas con la escritura e identificar las condiciones de la experiencia, pero no como una forma de patologización de los aprendizajes y prácticas de los niños y el maestro. 
que haya manifestaciones de verdad y a que se convoquen algunos acercamientos hacia el conocimiento de sí mismo. Por último, el cuento colectivo permite también que haya un tránsito a otra de las técnicas griegas como es la lectura, en la que el proceso lector permite que exista la reescritura con la que el niño identifica lo que le hace falta al texto, lo que no se entiende, lo que no es claro en los argumentos. De esta manera, la reescritura es un proceso que se confronta en el cuento colectivo.

El segundo ejercicio, el diario de experiencia, también se presenta en espacios de libertad que, al igual que en el ejercicio anterior, resignifica el lugar del niño-escritor bajo el dominio y conocimiento de sí mismo. Aquí la importancia de este diario es recurrir a la experiencia, que no se define como cualquier acontecimiento, sino como uno en el que el sujeto sale transformado y realmente atraviesa y modifica su existencia, de alguna manera. Por esta razón, se habla de esa experiencia del niño-escritor y cómo cuenta su experiencia y la escribe. Así mismo, se denotan las cuatro dimensiones del cuidado de sí - la inquietud, el cuidado, la preocupación y la ocupación- a partir del recuerdo, la memoria y el olvido, donde el recuerdo es una de las técnicas que también permite regresar y hacer una retrospectiva, reflexionar sobre lo que se ha hecho y traer esa experiencia. El recuerdo también denota una posibilidad de origen del acto creador, pues a partir del mismo y de la experiencia que se vuelve a vivir se da paso a la creación.

En el caso de la autobiografía, se manejan nuevamente las cuatro dimensiones del cuidado de sí: la inquietud, el qué escribir; el cuidado, el texto va a ser presentado a otra persona; la ocupación de cómo el estudiante construye su autobiografía con lo que sabe: y la preocupación por el qué escribir y por la identificación de alguna información o conocimiento exacto que el estudiante no tenga de sí mismo. La técnica de la meditación es otra de las técnicas que se presenta en la autobiografía, así como el recuerdo, la memoria y el olvido. Aquí el olvido toma una connotación importante ya que, en ocasiones, a los ejercicios les falta alguna información específica, entonces el olvido no se ve como un obstáculo, sino por el contrario potencia la inquietud, por medio de la cual se busca y se soluciona esa faltante de información. 
La autobiografía recurre a la experiencia como eso que atraviesa al sujeto y que lo modifica. Luego, a partir de un proceso de subjetivación, el autor escribe y moviliza un discurso, que bien puede estar conformado por verdades reproducidas, verdades modificadas o verdades que, en el mismo momento, construye, a partir de la escritura. Siempre parte de sí mismo, desde un proceso reflexivo y desde una obra que convoca a la reflexión y al conocimiento.

El autorretrato representa la estética de la existencia, ya que se toma la vida como el objeto. El niño se preocupa por simbolizarse y lograr delinear una imagen de sí mismo; por representase, de tal manera que haya formas de pensarse y verse organizadas y dirigidas para el intercambio con el compañero, de modo que se pueda resolver la pregunta sobre si realmente lo que está dibujado se parece o no a la persona. En este autorretrato hay un desencuentro, en la medida en la que el niño piensa lo que escribe, pero así mismo, piensa cómo se ve, entonces es un desencuentro que no se sabe dónde inicia, ya que las dos opciones son dirigidas al conocimiento del sujeto, pero en el momento de escribir, de llevar al papel, no existe un punto inicial. Se cuestiona, entonces, cuál sería el orden de la escritura. Nuevamente, se observa la experiencia como transformación y el reconocimiento del sujeto, en el recuerdo y en la memoria como en la emergencia del acto creador.

Por último está la correspondencia, ejercicio griego, en el que la escritura de cartas y su entrega se relacionaban con la amistad y con la raíz propia del humanismo. Este ejercicio se define desde el estilo de vida, ya que en la misma producción de cartas, los niños se preguntaban si lo que allí graficaban o lo que allí describían iba a tener algún impacto o algún efecto en el otro, de manera que se habla del cuidado del otro. Después de este ejercicio se realizó la práctica de escucha y lectura donde se implementaron acciones que permitieran vivir el silencio, la disposición y atención. También, se presentó la reescritura de las cartas, en la medida en la que algunos no entendían lo que estaba escrito allí, entonces, los mismos niños autores volvían a manifestar qué era lo que estaba escrito. En la correspondencia se manifestaron las verdades y el conocimiento del otro, pues a partir de la escritura de las cartas por los niños autores, se compartieron informaciones que solo se conocen entre amigos, por ejemplo, a través de los gustos o a manera de consejo se exponía aquello que podrían cambiar de sí, para mejorar la relación con los otros compañeros. 
Respecto a los resultados, se observa que en los ejercicios de escritura existe una transgresión del lenguaje cuya implicación consiste en que el sujeto "se oponga a la costumbre y a lo acordado culturalmente, por eso el autor se juega en lo escrito e invade los pactos, los mecanismos constituidos y los tratados apostando por alcanzar objetivos específicos e intereses de sí mismo" (Lara \& Pulido, 2017, pp. 197-198). Lo anterior se convierte en un lugar en el que el autor se permite la movilización y el cambio de las maneras cómo escribe, pues existe ese deseo por escribir y contar, desde el mismo sujeto, lo que lo motiva a escribir. De igual manera, se da una descolocación del sujeto y una retrospectiva de vida que lo impulsa a la escritura y al lugar de la pregunta, tan importante en todos los ejercicios que deviene en eje fundamental al inicio de cada ejercicio.

Así mismo, se reconoce la existencia de temas que se privilegian a la hora de escribir como: labores de campo, los animales, el cuidado de la naturaleza, experiencias de la escuela, los amigos, los deportes que realizan. Estos son temas que impulsan la escritura, como también el acercamiento con el entorno, ya que se trata de la escuela rural. Actividades vinculadas a la agricultura, a la ganadería, al cuidado del espacio, de igual modo, son representativas en los ejercicios de escritura, como lo es la escritura de sí que se realiza durante un tiempo indeterminado, porque los niños, en esos momentos, no estuvieron bajo la presión de un tiempo. También se distancian de la escolaridad porque llevan la escritura de sí, a otros escenarios, tales como campamentos filosóficos, a los que asisten o inclusive a la casa, donde, a veces, no llevan tareas o el trabajo que hacen en casa, sin embargo en estas ocasiones sí destinaron unos espacios para la escritura de sí.

También, se ve la vinculación de la escritura y la lectura como procesos cíclicos que representan e identifican las construcciones de sí mismo con el otro. Por último, la escritura tuvo una dimensión trascendental, al ubicar al niño autor desde los desplazamientos y la reestructuración de verdades, de manifestaciones de verdad. Todo ello ocupa otro lugar, en cuanto a la resignificación del ámbito escolar, en donde ya la escritura no se limitó a los aspectos operativos o automáticos, sino que se pensó hacia la obra, la creación, la reflexión y hacia las obras inspiradas, a partir de sentimientos, emociones y pensamientos. 
Para finalizar, se encuentra la escritura de sí, entre formas y modos y con la tendencia a pensar en ella, desde el lenguaje, en el que la escritura bordea límites, vórtices y transgrede otros espacios diferentes a los que habitualmente está acostumbrada a mostrarse. Se piensa entonces en una representación de la realidad y en una manifestación de verdad en una fotografía como producto de otros proyectos; la creación de guiones para obras de teatro en la que se manifiesten los temas con los que ya habían tenido acercamientos y donde existen otras formas de escribir; la inserción en lo gráfico, (allí los niños colocan en sus escritos algo de dibujo, algo de gráfico y eso los motiva mucho); una escritura centrada en lo gracioso y que recurre al uso de figuras retóricas, especialmente asociadas con las coplas, caligramas y rimas, con las que sienten mayor apropiación de este tipo de escritura.

\section{Conclusiones}

La escritura de sí dialoga con el sujeto, indaga por la constitución del sujeto y da apertura a la invención, puesto que el hecho de pensar y cuestionar al sujeto escritor implica volver la mirada sobre sí. La preocupación por el sujeto es la que promueve el conocimiento de su historia y su presente, así como el cuestionamiento por sus comportamientos, conductas y el reconocimiento de las relaciones que teje consigo mismo, con los demás y con el entorno, a través de sus escritos. Por esta razón, el sujeto se interroga y a través de sus expresiones y representaciones logra dar respuesta al encuentro de su interioridad. En tal reconocimiento él se identifica como el sujeto de la experiencia, se transforma y, gracias a la escritura que habita en espacios que se refractan sobre sí, el sujeto se impulsa a pensar de otro modo.

Lapregunta por el sujeto escritor, en la escuelay, específicamente, en los procesos de enseñanza y aprendizaje de la escritura, es una manera de considerar este objetivo fundamental de la formación desde otra mirada, la que se contempla desde el estudiante, desde su preocupación por conocerse y pensarse. Igualmente, se busca que en la escritura se encuentre la técnica que invite a los otros a hacer parte de la construcción de la vida y se compartan actitudes, ideas y preguntas, a partir de lo que se lea y se escuche con el otro. Por último, también se pretende que la escritura detone en invención y creación, acto que signifique para el sujeto la necesidad de explorar 
y verse reflejado en el intento de responder a la pregunta acerca del sujeto escritor y su constitución como tal.

Las entrevistas y el contacto con los niños permitieron conocer las diferentes concepciones que se crearon después de los ejercicios de escritura, una práctica que permite cambiar, modificar, repensar los otros efectos que va a tener el ejercicio, tanto a nivel escolar como a nivel individual. Los escenarios formativos permitieron la identificación de elementos con los cuales el niño autor se siente cercano a los procesos de subjetivación, que se potenciaron en espacios de reflexión entre el docente, investigador y el niño, en las relaciones del cuidado de sí y de lo otro.

Los ejercicios privilegiaron la creación y la invención como experiencias que permiten al niño hablar de sí mismo, narrarse y leerse de otras maneras. Además se piensa que la escritura de sí para la enseñanza es viable, ya que recurre a otros ámbitos donde puede detonar mayor acercamiento. La modificación de escenarios también es viable y vital en el cambio de concepción de escritura. La escritura de sí como ejercicio se profundizó con las otras técnicas griegas, la escucha, la lectura. Estas tres técnicas son complementarias en la reflexión y necesarias para el desarrollo del ejercicio de la narración y de la lectura de sí mismo. Por último, se considera necesario el pensar la escritura de sí como práctica y técnica que debe ser continua, puesto que en la medida en que haya un entrenamiento del mismo ejercicio, se logra una mejoría. En este caso, se debe a los acercamientos y a la formación de un hábito que fortalece el conocimiento y la perfección del sujeto, en la estimación de que los niños son autores de su estar en el mundo.

\section{Referencias}

Aguilar, S. (2017). La práctica de la escritura en Foucault: literatura, locura, muerte y escritura de sí. Dorsal. Revista De Estudios Foucaultianos, (2), 219-244. http://dx.doi.org/10.5281/zenodo.804766

Aquino, J. (2011). A escrita como modo de vida: conexões e desdobramentos educacionais. Educação e Pesquisa, 37(3), 641-656. https://dx.doi. org/10.1590/S1517-97022011000300013

Arias, M. (2012). La escritura como dispositivo constituyente de sujeto. Hallazgos, 9(18), 67-77. Recuperado de: https://revistas.usantotomas. edu.co/index.php/hallazgos/article/view/720 
Castorina, R. (2013). Pensare e vivere il 'fuori'. Etopolitica, ontologia e scrittura nella riflessione di Michel Foucault. Lo Sguardo (11), 173-197

Cely, A., Espinosa, I., \& Suárez, M. (2016). Filosofía y literatura: Una experiencia de encuentro con la infancia. Quaestiones Disputatae, 9 (19), 28-43

Delory-Momberger, C. (2006). Formação e socialização: os ateliês biográficos de projeto. Educação e pesquisa, 32 (2), 359-371 http:// dx.doi.org/10.1590/S1517-97022006000200011.

Demetrio, D. (2013). L'autobiografia come formazione e filosofia di vita. Per gli individui o per le persone?. Lo Sguardo (11), 371-381.

Deleuze, G. (1993). La literatura y la vida. En Crítica y Clínica. Barcelona: Anagrama

Domingues, M. (2004). O desafio ético da escrita. Psicologia \& Sociedade; $16(1), 146-150$.

Espinel, O., \& Pulido, O. (2017a). La enseñanza de la filosofía. Entre experiencia filosófica y ensayo. Universithas Philoshophica, 34(69), 121-142. https://doi.org/10.11144/Javeriana.uph34-69.efee

Espinel, O., \& Pulido, O. (2017b). Perspectivas metodológicas en el "último" Foucault. Nuevos horizontes, nuevos problemas. En O. Pulido, \& O. Espinel., Formas y expresiones metodológicas en el último Foucault. Tunja: Editorial Uptc.

Foucault, M. (1998). ¿Qué es un autor? Córdoba: Litoral.

Foucault, M. (1999). Estética, ética y hermenéutica. Barcelona: Paidós

Foucault, M. (2002). La hermenéutica del sujeto. Curso en el College de France (1981-1982). México: Fondo de Cultura Económica.

Foucault, M. (2010). El coraje de la verdad. Buenos Aires: FCE

Foucault, M. (2012a). Un peligro que seduce. Valladolid, España: Cuatro ediciones.

Foucault, M. (2012b). Historia de la sexualidad II: el uso de los placeres. Madrid: Biblioteca Nueva

Foucault, M. (2013). La inquietud por la verdad: Escritos sobre la sexualidad y el sujeto. Buenos Aires: Siglo Veintiuno Editores

Foucault, M. (2015). La genealogía de la ética. En La ética del pensamiento: para una crítica de lo que somos (pp. 343-369). Madrid: Biblioteca Nueva. 
Hadot, P. (2006). Ejercicios Espirituales y filosofía antigua. España: Siruela

Hernández, A., \& Hernández, L. (2012). Ecosistemas escolares para el desarrollo del pensamiento creativo en los niños. Praxis \& Saber, 3(6), 141-164. https://doi.org/10.19053/22160159.2007

Lara, P. (2017). Ética, verdad y cuidado de sí: aproximaciones al último Foucault. En O. Pulido, \& O. Espinel., Formas y expresiones metodológicas en el último Foucault. Tunja: Editorial Uptc.

Lara, P., \& Pulido, O. (2017). Sujeto, distancia y muerte: a propósito de Foucault y la escritura. En O. Pulido, M. Suárez \& O. Espinel., Pensar de otro modo. Herramientas filosóficas para investigar en educación. Tunja: Editorial Uptc.

Lechuga, G. (2007). Breve introducción al pensamiento de Foucault. México: Universidad Autónoma Metropolitana.

Lluch, E. (2013) Foucault: la escritura como experiencia de transformación. Lo Sguardo, (11), 199-211.

López, R. (2015). Escribir para cuidar de sí y del otro: pensarse, crearse y regirse desde la escritura. Actualidades Pedagógicas, (65), 229-244. https://doi.org/10.19052/ap.3491

Loponte, L. (2006). Escritas de si (e para os outros) na decência em arte. Educação (UFSM), 31(2), 295-304. http://dx.doi. org/10.5902/19846444.

Lucci, A. (2013). «E fuor dell'opera si è capolavoro». Sull'autobiografia, a partire da Peter Sloterdijk. Lo Sguardo (11), 299-313

Morales, L., \& Pulido, O. (2019). Ambientes filosóficos para la lectura en la escuela rural. Praxis \& Saber, 9(21), 99-124. https://doi. org/10.19053/22160159.v9.n21.2018.8925

Morresi, Z. (2005). La operatividad del discurso en la construcción de subjetividades. La Trama de la Comunicación, 10, 1-8. Recuperado de: https://www.redalyc.org/pdf/3239/323927060001.pdf

Pérez, S. (2006). La travesía de la escritura. Taurus: México.

Pulido, O. (2017). Apuntes y reflexiones para pensar a Foucault de otro modo: consideraciones metodológicas de una filosofía de los relámpagos. En O. Pulido, \& O. Espinel., Formas y expresiones metodológicas en el último Foucault. Tunja: Editorial Uptc.

Pulido, C., Suárez, V., \& Espinel, B. (2017). Pensar de otro modo. Herramientas filosóficas para investigar en educación. Tunja: Editorial Uptc. 
Rodari, G. (1973). Gramática de la fantasía. Buenos Aires: Editorial Argos Vergara, S. A

Scholze, L. (2007). Narrativas de si e a estética da existencia. Aberto, 21(77), 61-72

Schmid, W. (2002). En busca de un nuevo arte de vivir. La pregunta por el fundamento y la nueva fundamentación de la ética en Foucault. Valencia: Pre-Textos

Silveira, L., Nascimento do, M., \& Galindo, D. (2016). Escrita, psicologia e produção de cuidado: ética, estética e política. Arquivos Brasileiros de Psicologia, 68(1). 1-11. Recuperado de: https://www.redalyc.org/ articulo.oa?id $=229046737008$

Suárez, M., Mariño, L., \& Espinel, O. (2017). Filosofía e infancia: un proyecto para cuidar de sí. En O. Pulido, M. Suárez, O. Espinel., Pensar de otro modo. Herramientas filosóficas para investigar en educación. Tunja: Editorial Uptc. 